\title{
Safety of fibreoptic bronchoscopy in asthmatic and control subjects and effect on asthma control over two weeks
}

\author{
M Humbert, D S Robinson, B Assoufi, A B Kay, S R Durham
}

\begin{abstract}
Background - Concerns remain about the safety of bronchoscopy in asthma and there are few data on the effect of this procedure on asthma control in the days or weeks following bronchoscopy.

Methods - In an initial study of bronchoalveolar lavage and bronchial biopsies in asthmatic and control subjects, data on peak expiratory flow rates (PEFR) collected prospectively before and after the procedure were available from 21 of the 29 asthmatic subjects studied. These showed a median 23\% fall in PEFR from baseline after bronchoscopy (range 3-58\%). To determine whether this fall in PEFR following bronchoscopy reflected bronchospasm or the effect of sedation, PEFR and spirometric tests were performed during the two hours following bronchoscopy in a further study of 15 symptomatic asthmatic subjects and 20 non-asthmatic controls. To examine the effect on asthma control, asthmatic patients recorded PEFR, symptom scores, and medication use for two weeks before and after bronchoscopy.
\end{abstract}

Results - After bronchoscopy with bronchial biopsies there was no difference between the median maximal fall in either PEFR or arterial oxygen saturation between the 15 asthmatic patients $(10.4 \%$ and $4 \%$, respectively) and 20 controls (12\% and $3 \%$ ). Moreover, there were no significant changes in PEFR, symptom score, or medication use by the asthmatic subjects in the two weeks after bronchoscopy when compared with the two weeks before bronchoscopy.

Conclusions - Fibreoptic bronchoscopy is well tolerated in asthmatic subjects.

Department of Allergy and Clinical

Immunology,

National Heart and

Lung Institute,

Dovehouse Street,

London SW3 6LY, UK

M Humbert

D S Robinson

B Assoufi

A B Kay

S R Durham

Correspondence to: Dr S R Durham.

Received 7 July 1995

Returned to authors

20 November 1995

Revised version received

Revised version rece

10 January 1996

26 January 1996
The use of bronchoalveolar lavage and bronchial biopsy specimens has led to substantial asthma control.

(Thorax 1996;51:664-669)

Keywords: asthma, bronchoscopy, safety. advances in our understanding of the pathogenesis of asthma. ${ }^{1-5}$ Despite initial concerns regarding risks of bronchospasm, laryngospasm, or hypoxaemia, ${ }^{6-8}$ the technique is now widely used in asthma research. Two National Heart, Lung and Blood Institute workshops have suggested guidelines for investigational use of bronchoscopy in asthma, ${ }^{910}$ and the 1990 guidelines stated that premedication with bronchodilators and atropine could be used or omitted, depending on the specific research protocol and the safety and comfort of the individual subject. ${ }^{10}$ It was stressed that careful clinical monitoring should include arterial haemoglobin oxygen saturation during the procedure, and peak expiratory flow rate (PEFR) and forced expiratory volume in one second $\left(\mathrm{FEV}_{1}\right)$ at $15-30$ minute intervals until the condition of the patient is stable.

Djukanovic et al demonstrated in a prospective study that bronchoscopy with bronchoalveolar lavage and bronchial biopsy was well tolerated by subjects with asthma, ${ }^{11}$ and van Vyve et al reported no significant adverse effects after bronchoscopy without premedication with a bronchodilator even in severe asthmatics with a low $\mathrm{FEV}_{1}{ }^{12}$ Most authors have not detected any significant effect of fibreoptic bronchoscopy on bronchial hyperresponsiveness in subjects with asthma, ${ }^{1113-15}$ although one study showed increased responsiveness. ${ }^{16}$ No data are available on the effect of fibreoptic bronchoscopy on asthma control in the weeks following bronchoscopy.

In an initial study involving bronchoalveolar lavage and bronchial biopsies in 29 asthmatic and control subjects ${ }^{17}$ we prospectively monitored PEFR after the procedure. Here we report data from 21 of the 29 asthmatic subjects in this study from whom data were available, in whom a fall in PEFR was found to be associated with bronchoscopy. We went on to compare the effects of bronchoscopy including the collection of 10 bronchial biopsy specimens in a further 15 asthmatic and 20 control subjects in a protocol including sedation with intravenous midazolam and premedication with nebulised salbutamol in accordance with published guidelines. Changes in the asthmatic subjects were compared with those in normal control subjects in order to detect immediate effects of sedation (as opposed to bronchospasm) on peak flow rates and $\mathrm{FEV}_{1}$. We have also documented the effect of bronchoscopy on PEFR, asthma symptoms, and bronchodilator requirements in the two weeks after the procedure. 
Table 1 Daily asthma symptom score (DASS)

\begin{tabular}{ll}
\hline (no symptoms) & $\begin{array}{l}\text { Sleep: Woke once because of asthma but able to go back to sleep } \\
\text { Other symptoms: Day time symptoms from time to time which } \\
\text { did not interfere with daily routine }\end{array}$ \\
2 (moderate symptoms) & $\begin{array}{l}\text { Sleep: Woke more than once } \\
\text { Other symptoms: Day time symptoms that interfered with daily } \\
\text { routine noticed often } \\
\text { Sleep: Had great difficulty sleeping }\end{array}$ \\
Other symptoms: Day time symptoms present for most of the \\
day with radically changed daily routine
\end{tabular}

The daily asthma score (DASS) is the sum of the scores 0-3 given each day for sleep quality as above (sleep), and for each of chest tightness, wheezing, cough, sputum production, and dyspnoea (other symptoms) graded as above. Maximum possible score for one day is 18 .

\section{Methods}

STUDY DESIGN

Data are presented from two separate study periods. In the first study 29 asthmatic subjects and 13 normal volunteers underwent bronchoscopy with bronchoalveolar lavage and up to four endobronchial biopsy specimens between 1990 and 1991. The characteristics of these subjects, the inclusion criteria, and the bronchoscopy protocol have been described previously. ${ }^{17}$ After bronchoscopy the PEFR values were recorded prospectively in 21 of the 29 asthmatic subjects (data collection was started after the first eight subjects had been included) and, on the basis of an observed fall in PEFR, we went on to collect data in a second study in which 15 asthmatic subjects and 20 nonasthmatic control subjects underwent fibreoptic bronchoscopy and up to 10 endobronchial biopsies. Bronchoalveolar lavage was not performed in the second study protocol which was designed to gather bronchial biopsy specimens from asthmatic subjects and atopic and non-atopic controls for study of local cytokine gene expression.

In the second study asthma was defined on the basis of a clear clinical history, and evidence of more than $20 \%$ reversibility of $\mathrm{FEV}_{1}$ or PEFR either spontaneously or after inhaled $\beta_{2}$ agonist. Asthmatic subjects had current symptoms and an inhaled histamine concentration of a maximum of $6 \mathrm{mg} / \mathrm{ml}$ was required to induce a $20 \%$ fall in $\mathrm{FEV}_{1}\left(\mathrm{PC}_{20}\right)$ in the previous two weeks. Atopy was defined as one or more positive skin prick tests (weal diameter of $3 \mathrm{~mm}$ more than the negative control, ALK, Horsholm, Denmark) to one or more of house dust

Table 2 Patient information

\begin{tabular}{lcc}
\hline Study 1: & & \\
No. of asthmatics & 21 & \\
No. symptomatic & 14 & \\
Age & $25 \cdot 3(18-32)$ & \\
FEV $_{1}(\%)$ & $87 \cdot 5(62-129)$ & \\
PEFR (\%) & $96(67-121)$ & \\
Methacholine PC $_{20}$ & $1 \cdot 57(0 \cdot 24-15.5)$ & \\
& & Controls \\
Study 2: & & 20 \\
No. of subjects & Asthmatics & $15 / 5$ \\
M/F & 15 & $10 / 10$ \\
Atopics/non-atopics & $9 / 6$ & $68(3-353)$ \\
Serum IgE (IU/ml) & $12 / 3$ & $0 \cdot 1(0 \cdot 0-0 \cdot 4)$ \\
Blood eosinophils $\left(10^{9} / 1\right)$ & $180(29-3749)$ & $106(93-123)$ \\
PEFR (\%) & $0 \cdot 3(0 \cdot 1-0 \cdot 6)$ & $101(81-123)$ \\
FEV $(\%)$ & $90(73-113)$ & $100(88-129)$ \\
FVC (\%) & $87(59-106)$ & $>16$ \\
Histamine PC $_{20}$ & $100(81-118)$ & N/A \\
DASS & $0 \cdot 69(0 \cdot 02-6 \cdot 0)$ & \\
\hline
\end{tabular}

Data are medians with range except for $\mathrm{PC}_{20}$ (geometric mean).

$\mathrm{PEFR}=$ peak expiratory flow rate; $\mathrm{FEV}_{1}=$ forced expiratory volume in one second; $\mathrm{FVC}=$ forced vital capacity; DASS = daily asthma symptom score (see table 1 ). mite, mixed grass pollens, mixed moulds, cat, and dog. Non-atopic control subjects were defined by negative skin prick tests, total serum IgE levels less than $150 \mathrm{U} / \mathrm{ml}$, and negative radioallergosorbent test (RAST) for specific IgE to 25 aeroallergens (Pharmacia Diagnostics, Uppsala, Sweden). Atopic control subjects had no symptoms of asthma, normal lung function, and less than $20 \%$ reversibility in $\mathrm{FEV}_{1}$ or PEFR, with histamine $\mathrm{PC}_{20}$ of more than $16 \mathrm{mg} / \mathrm{ml}$. Full clinical examination and spirometric tests (Vitalograph, Buckinghamshire, UK) at baseline and after $\beta$ agonist inhalation (salbutamol $200 \mathrm{~g}$ by metered dose inhaler), histamine $\mathrm{PC}_{20}$, skin prick tests, chest radiography, full blood counts, total serum $\mathrm{IgE}$, and RAST were performed in all patients and control subjects.

Asthma symptoms were assessed daily and recorded on a diary card for two weeks before and after the bronchoscopy using a daily symptom score which assessed sleep, chest tightness, wheeze, cough, sputum production, and dyspnoea (DASS, table 1), rescue $\beta$ agonist use, and morning and evening PEFR as the best of three prebronchodilator readings.

Both studies were approved by the ethics committee of the Royal Brompton Hospital.

\section{PATIENTS}

Data on PEFR following bronchoscopy were collected from 21 asthmatic subjects studied in the first protocol. Clinical data on these subjects are presented in table 2 .

Fifteen asthmatic and 20 control subjects were included in the second study between April and October 1994. Patient details are shown in table 2 .

In both studies none of the subjects had received oral corticosteroids in the last three months, and none had received inhaled corticosteroids for three months in the first study and two weeks before the second study. None of the subjects studied was a current smoker or had smoked for more than five pack years in the past. Exclusions in the second study group were age less than 18 or more than 55 years, $\mathrm{FEV}_{1}<50 \%$ predicted, acute or chronic infection, pregnancy, breast feeding, or any other significant medical condition.

\section{FIBREOPTIC BRONCHOSCOPY}

In both studies fibreoptic bronchoscopy was performed using an Olympus BFP20 bronchoscope with $2 \cdot 2 \mathrm{~mm}$ biopsy channel (Olympus Corporation, Tokyo, Japan). Subjects were admitted to the day ward having fasted for at least four hours, and baseline pulse, blood pressure, and PEFR were recorded. In the second study spirometric tests were also performed. Asthmatic and control subjects were given salbutamol $2.5 \mathrm{mg}$ by nebuliser $10-15$ minutes before the bronchoscopic examination. In the second study PEFR and spirometric tests were then repeated. Immediately before the procedure atropine $0.5 \mathrm{mg}$ was given by slow intravenous injection, and midazolam sedation 5-10 mg was also given intravenously. 
The nose and throat were anaesthetised with $1 \%$ lignocaine spray and gel, the bronchoscope was gently introduced, and the vocal cords were anaesthetised with $4 \%$ lignocaine spray. The bronchial tree was anaesthetised with $2 \%$ lignocaine spray. Supplemental oxygen was given throughout the procedure via a nasal cannula, and oxygenation was monitored by finger probe pulse oximetry (Ohmeda, Wallingford, Connecticut, USA).

In the first study, after inspection of the bronchial tree, bronchoalveolar lavage fluid was obtained from either the right middle lobe or lingula by instillation of four $60 \mathrm{ml}$ aliquots of warmed $\mathrm{pH}$ adjusted $0.9 \%$ saline ( $240 \mathrm{ml}$ total) which were collected by gentle machine suction. This was followed by up to four endobronchial biopsy specimens from the segmental and subsegmental carinae of the same lung, obtained using Olympus FB15C forceps.

In the second study up to 10 endobronchial biopsy specimens were obtained using FB15C forceps. No bronchoalveolar lavage was performed.

All subjects were assessed clinically immediately after the bronchoscopic examination and PEFR and spirometric values were recorded before administration of a further $2.5 \mathrm{mg}$ nebulised salbutamol. Subjects were then observed on the ward for at least two hours with PEFR (first study) and PEFR and spirometry (second study) every 30 minutes. After two hours subjects were assessed clinically by a physician and, if stable, were discharged home. All subjects were given an emergency contact telephone number and a follow up appointment.

\section{STATISTICAL ANALYSIS}

Data are presented as medians with range. Comparison between time points or groups was by the Mann-Whitney U test with Bonneferoni's correction for multiple comparisons where appropriate. The daily asthma symptom score, medication use, and morning PEFR for each day over the two weeks before and after the bronchoscopic examination were analysed by analysis of variance (ANOVA) to detect significantly different time points for the asthmatic subjects in the second study. Comparison between the maximal fall in PEFR in asthmatic subjects included in study 1 (bronchoalveolar lavage and bronchial biopsy specimens) and study 2 (bronchial biopsy specimens only) was by the Mann-Whitney U test. Correlations between the fall in PEFR associated with bronchoscopy and baseline $\mathrm{FEV}_{1}$ and bronchial responsiveness in both study groups were sought using Spearman's rank correlation.

\section{Results}

Fibreoptic bronchoscopy was well tolerated. Two subjects in the first study and one asthmatic subject in the second study had mild symptomatic wheeze after the bronchoscopic examination which was rapidly reversed with an additional dose of salbutamol by nebuliser.
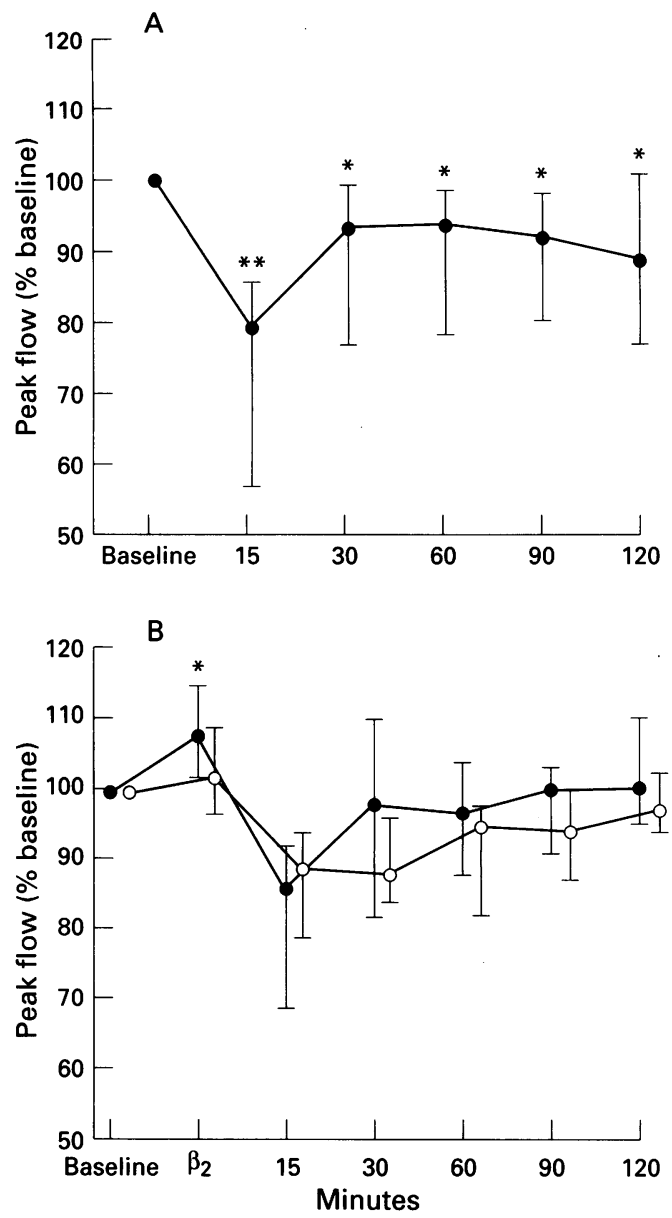

Figure 1 Peak expiratory flow rate (\% predicted) before and at 15, 30,60,90, and 120 minutes after fibreoptic bronchoscopy in $(A) 21$ atopic asthmatic subjects and $(B)$ 15 asthmatic subjects (O) and 20 non-asthmatic controls (O). Peak flow rates after administration of a $\beta$ agonist by nebuliser are also shown in $(B)$. In $(A)$ peak flow rates after bronchoscopy are compared with baseline values by the Mann-Whitney $U$ test using the Bonneferoni correction for multiple comparisons $\left({ }^{* *} p<0 \cdot 01 ;{ }^{*} p<0.05\right)$. In (B) peak flow rates in asthmatic subjects and controls are compared by the Mann-Whitney $U$ test $\left({ }^{*} p<0 \cdot 05\right)$. Medians with interquartile ranges are shown.

In the first study there was a significant fall in PEFR after fibreoptic bronchoscopy in 21 asthmatic subjects (fig 1 ).

The second study also showed a significant fall in PEFR in asthmatic subjects after fibreoptic bronchoscopy, but this was also seen in non-asthmatic control subjects with no significant difference between the groups (fig 1). Peak expiratory flow rates, $\mathrm{FEV}_{1}$, and forced vital capacity (FVC) were not significantly different from baseline at two hours after bronchoscopy (table 3). Oxygen saturation also showed a fall during bronchoscopic examination in both asthmatic and control subjects (table 3). Supplemental oxygen was given throughout the procedure in all cases at a rate of $6 \mathrm{l} / \mathrm{min}$, and no bronchoscopic examination was interrupted because of arterial oxygen desaturation.

In study 1 there was a significant relationship between both airways hyperresponsiveness and $\mathrm{FEV}_{1}$ at baseline and the maximal fall in PEFR associated with fibreoptic bronchoscopy amongst the 21 asthmatic subjects studied ( $r=$ 
Table 3 Peak expiratory flow rate (PEFR), forced expiratory volume in one second $\left(F E V_{1}\right)$, and forced vital capacity $(F V C)$ as percentage of prebronchoscopy values, and oxygen saturation measurements two hours after bronchoscopy in 15 asthmatic subjects and 20 controls (study 2 population)

\begin{tabular}{lcr}
\hline & \multicolumn{1}{c}{ Asthmatics } & \multicolumn{1}{c}{ Control subjects } \\
\hline PEFR 2 hours after bronchoscopy (\% baseline) & $100(85-122)$ & $97(85-103)$ \\
FEV $1_{\text {2 hours after bronchoscopy (\% baseline) }}$ & $105(89-126)$ & $100(90-110)$ \\
FVC 2 hours after bronchoscopy (\% baseline) & $99(93-115)$ & $99(91-107)$ \\
$\begin{array}{l}\text { Prebronchoscopy oxygen saturation (\%) } \\
\text { (all with supplemental oxygen) }\end{array}$ & $100(99-100)$ & $100($ all 100) \\
Minmum prebronchoscopy SaO $_{2}(\%)$ & $96(88-98)$ & $97(91-99)$ \\
\hline
\end{tabular}

-0.55 between percentage fall in PEFR and $\mathrm{PC}_{20}(\mathrm{p}<0.05)$ and $r=0.54$ with baseline $\mathrm{FEV}_{1}$ $(\mathrm{p}<0.05))$. In study 2 the relationship between baseline $\mathrm{FEV}_{1}$ and fall in PEFR amongst the 15 asthmatic subjects studied was not significant and there was no association between $\mathrm{PC}_{20}$ and fall in PEFR. The median maximal percentage fall in PEFR associated with a bronchoscopy protocol including both bronchoalveolar lavage and bronchial biopsies was $23.0 \%$, whilst that associated with the bronchoscopy protocol in study 2 (bronchial biopsy
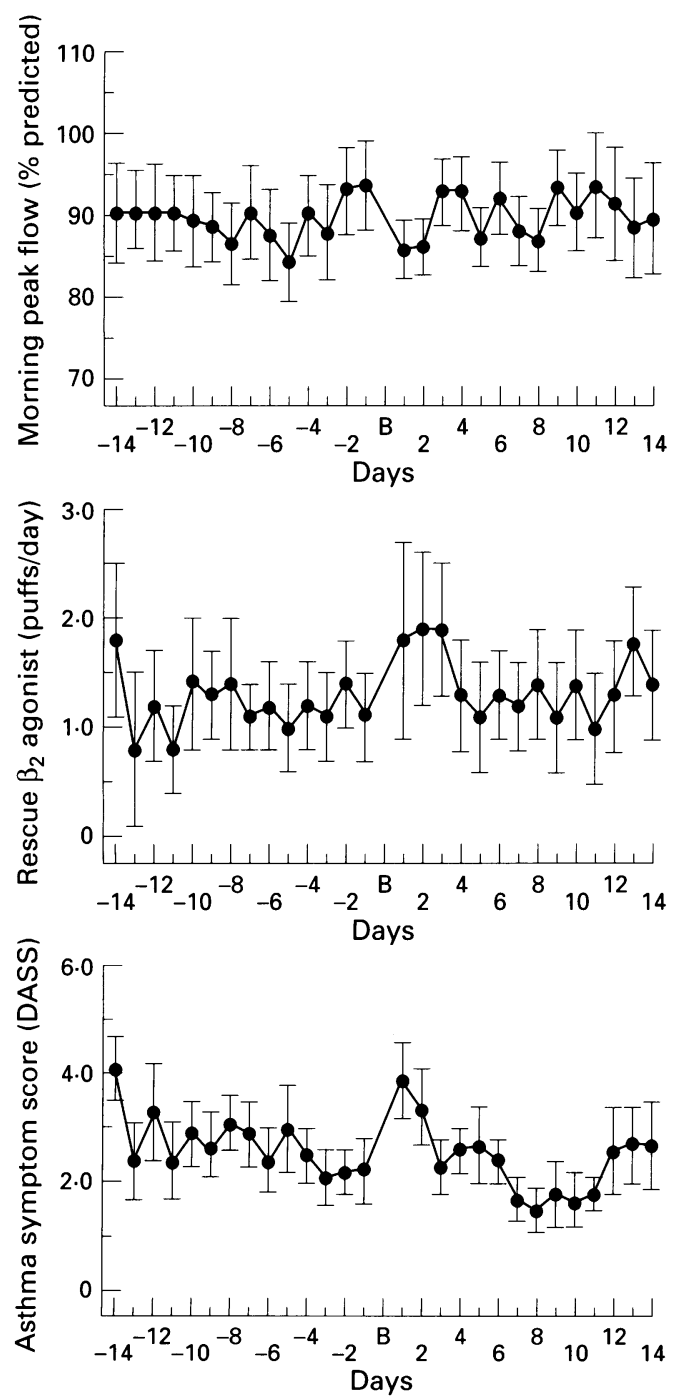

Figure 2 Morning peak flow rate (\% of predicted), $\beta$ agonist use (puffs from metered dose inhaler per day), and daily asthma score (DASS) in 14 asthmatic subjects for 14 days before and after fibreoptic bronchoscopy with bronchial biopsies (B). Means and standard errors are bronchial biopsies (B). Means and standard errors are
shown. No significant differences were detected between data from any of the 28 days by analysis of variance (ANOVA). specimens without bronchoalveolar lavage) was $10 \cdot 4 \%$, although this difference did not achieve statistical significance.

Asthmatic subjects recorded their daily asthma symptoms, rescue salbutamol therapy, and PEFR for two weeks before and after bronchoscopy. No significant change in these measures of asthma control was seen in the two weeks following bronchoscopy when compared with the two weeks before the procedure by ANOVA (fig 2).

\section{Discussion}

In this report of a total of 36 asthmatic subjects and 20 control volunteers no complications of fibreoptic bronchoscopy were observed. In all cases the procedure was clinically well tolerated. In the first study protocol involving bronchoalveolar lavage and bronchial biopsy specimens there was a significant fall in PEFR after bronchoscopy which reversed partly with nebulised salbutamol and was related to baseline airways obstruction and bronchial responsiveness. The second study protocol involving up to 10 bronchial biopsy specimens but no bronchoalveolar lavage showed a trend towards a smaller effect on PEFR than the protocol in study 1 which involved both biopsy specimens and bronchoalveolar lavage. There was no difference in the effect of the bronchoscopy on PEFR between asthmatic and control subjects, suggesting that falls in PEFR may reflect sedation rather than bronchospasm. Bronchoscopy had no significant effect on asthma symptoms, peak expiratory flow rates, or medication use in the two weeks following the procedure.

The first study showed a significant fall in PEFR in asthmatic subjects after bronchoscopy with bronchoalveolar lavage and bronchial biopsy. This was not completely reversed by $\beta$ agonists (fig 1) which suggests that it might reflect the effect of sedation on performance of forced manoeuvres in addition to bronchospasm induced by bronchoscopy. We examined this possibility by carefully documenting symptoms and PEFR in normal as well as asthmatic subjects in the second more detailed study of the safety of bronchoscopy in asthma. This showed that there was no difference between asthmatic and control subjects in the effect of fibreoptic bronchoscopy with biopsy alone on PEFR. Control subjects were given a nebulised $\beta$ agonist in this protocol to control for any effect on airway cell characteristics, but all controls had normal lung function and showed no evidence of bronchial hyperresponsiveness. It is therefore unlikely that any of the fall in PEFR seen in the control group is attributable to bronchospasm, but rather that it reflects the effect of sedation on the performance of voluntary forced expiratory manoeuvres. Thus, it seems likely that the fall in PEFR seen in asthmatic subjects also reflects sedation in addition to any bronchospasm induced by the procedure. Nonetheless, the relationship seen between airway calibre $\left(\mathrm{FEV}_{1}\right)$ and bronchial responsiveness $\left(\mathrm{PC}_{20}\right)$ at baseline and the maximal fall in PEFR after bronchoscopy in study 
1 suggests that some of the observed fall in PEFR may reflect bronchospasm related to asthma severity. This is in agreement with the findings of Djukanovic et al who also included bronchoalveolar lavage in their protocol. ${ }^{12}$ Two subjects in study 1 and one in study 2 required additional bronchodilator to that given before and after the bronchoscopy and all three showed rapid resolution of symptoms. The falls

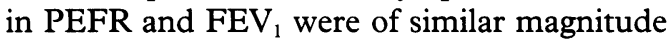
to those recorded after inhalation challenge of asthmatic subjects. ${ }^{18}$

In the absence of bronchodilator premedication van Vyve et al showed a greater fall in lung function in asthmatic subjects than in controls after bronchoscopy. ${ }^{12}$ In contrast to our findings, Djukanovic et al also found that bronchoscopy had a more marked effect on lung function in asthmatic than in control subjects, even when nebulised salbutamol was given before the procedure. ${ }^{11}$ The difference in our results may be explained by the lack of bronchoalveolar lavage in our second study, since Djukanovic et al performed both bronchoalveolar lavage and bronchial biopsies. In view of the trend to a greater fall in PEFR seen in study 1 (which included bronchoalveolar lavage) than in study 2 (which did not), it is possible that bronchoalveolar lavage may be more likely than bronchial biopsies to provoke bronchospasm in asthmatic subjects. A formal comparison in a single study would be required to confirm this. However, this may not be possible for ethical or scientific reasons. Baseline asthma severity was similar (in terms of $\mathrm{FEV}_{1}, \mathrm{PEFR}$, and bronchial responsiveness) between the asthmatic subjects in studies 1 and 2 , so the greater fall in PEFR in study 1 cannot be explained by differences in asthma severity. Oxygen desaturation during bronchoscopy was mild in our patient population receiving routine supplemental oxygen. We suggest that oxygen should be given to all subjects undergoing investigational bronchoscopy, and that $\beta$ agonists should be given to asthmatic subjects unless this will specifically interfere with cellular or mediator measurements.

The understanding of complex diseases such as asthma demands analysis of cellular and molecular aspects of the disease. Since bronchial biopsy specimens are small, multiple specimens may be needed if sufficient information is to be obtained. In the absence of clinical information, the 1990 expert workshop could not recommend taking more than six bronchial biopsy specimens. ${ }^{10}$ Our study suggests that, if bronchoalveolar lavage is not performed, harvesting up to 10 bronchial biopsy specimens even in patients with moderately severe asthma $\left(\mathrm{PC}_{20}<0.20 \mathrm{mg} / \mathrm{ml}\right)$ is well tolerated.

It is important to describe the risk factors for potential bronchoscopy-induced side effects in asthma. In agreement with Djukanovic et al we have shown a negative correlation between airways hyperresponsiveness and the percentage fall in PEFR after bronchoscopy when the procedure includes both bronchoalveolar lavage and bronchial biopsies. ${ }^{11}$ Thus, if bronchoalveolar lavage and bronchial biopsies are to be performed, airways limitation and bronchial responsiveness may predict the effects of bronchoscopy. However, in our second study of symptomatic asthmatic subjects with $\mathrm{PC}_{20}$ ranging from 0.02 to $6.0 \mathrm{mg} / \mathrm{ml}$ histamine we did not find such a correlation. This seems likely to reflect the lack of bronchoalveolar lavage in the second study protocol. Similarly, van Vyve et al found no difference between mild and severe asthmatic subjects in the fall in $\mathrm{FEV}_{1}$ induced by bronchoscopy. ${ }^{12}$

Our data on medium term safety of bronchoscopy in asthmatic subjects is novel. All but one of the previous reports have shown no significant effect of bronchoscopy on airways responsiveness in asthma. ${ }^{113-16}$ In addition, we have shown that asthma symptom scores, rescue salbutamol intake, and morning PEFR were not altered up to two weeks after the procedure. Occasional individual patients did show an increase in symptom score, PEFR variability, or $\beta$ agonist use in the two weeks after the procedure when compared with the run-in period, but some also showed an improvement in the measures which suggests that this may reflect the variable nature of asthma rather than an effect of bronchoscopy per se. Since the second study protocol did not include bronchoalveolar lavage, we cannot comment on whether longer term asthma control may be affected if both bronchoalveolar lavage and bronchial biopsies are performed.

These clinical data confirm and extend previous reports on the safety of bronchoscopy in asthma and support the view that bronchoscopy is well tolerated even in patients with moderately severe disease when the procedure is performed by trained persons. Previous prospective studies in asthma are small, and even rare complications in volunteers would be a cause for concern. Meticulous surveillance of all asthmatic volunteers who undergo bronchoscopy is therefore essential. Any side effect which occurs even in a very limited number of patients should be reported and safety guidelines should be updated regularly.

The authors acknowledge Dr AM Bentley for help with the initial bronchoscopy study, Mr D Cramer, Ms S Meah and staff of Lind Ward, Royal Brompton Hospital.

These studies were supported by the Medical Research Council (UK). MH was supported by a grant from the Société de Pneumologie de Langue Française.

1 Djukanovic R, Roche WR, Wilson JW, Beasley CRW, Twentyman OP, Howarth PH, et al. Mucosal inflammation in asthma. Am Rev Respir Dis 1990;142:434-57.

2 Smith DL, Deshazo RD. Bronchoalveolar lavage in asthma. Am Rev Respir Dis 1993;148:523-32.

3 Crump JW, Pueringer RJ, Hunninghake GW. BronchoCrump JW, Pueringer RJ, Hunninghake GW. Broncho-
alveolar lavage and lymphocytes in asthma. Eur Respir $\mathcal{f}$ 1991;4:39-46s.

4 Bousquet J, Chanez P, Lacoste JY, Barnéon G, Ghanavian $\mathrm{N}$, Enander I, et al. Eosinophilic inflammation in asthma. N, Enander I, et al. Eosinophilic infl

5 Robinson DS, Hamid Q, Sun Ying, Tsicopoulos A, Barkans J, Bentley AM, et al. Predominant Th2-like bronchoalveolar lavage T-lymphocytes population in atopic asthma. N Engl f Med 1992;326:298-304.

6 Rosenow EC, Andersen HA. Bronchoscopically induced bronchospasm. Chest 1976;70:565-6.

7 Albertini RE, Harell JH, Kurihara N, Moser KM. Arteria hypoxemia induced by fiberoptic bronchoscopy. $\mathcal{F} A M A$ 1974;230:1666-7.

8 Sahn SA, Scoggin C. Fiberoptic bronchoscopy in bronchia asthma: a word of caution. Chest 1976;69:39-42.

9 Summary and recommendations of a workshop on the investigative use of fiberoptic bronchoscopy and bronchoalveolar lavage in individuals with asthma. 7 Allergy Clin Immunol 1985;76:145-7. 
10 Workshop summary and guidelines: investigative use of bronchoscopy, lavage, and bronchial biopsies in asthma
and other airway diseases. $\mathcal{F}$ Allergy Clin Immunol 1991; and other

11 Djukanovic R, Wilson JW, Lai CKW, Holgate ST, Howarth PH. The safety aspects of fiberoptic bronchoscopy, bronchoalveolar lavage, and endobronchial biopsy in asthma. Am Rev Respir Dis 1991;143:772-7.

12 Van Vyve T, Chanez P, Bousquet J, Lacoste JY, Michel F-B, Godard P. Safety of bronchoalveolar lavage and bronchial biopsies in patients with asthma of variable severity. Am Rev Respir Dis 1992;146:116-21.

13 Kirby JG, O’Byrne PM, Hargreave FE. Bronchoalveolar lavage does not alter airway responsiveness in asthmatic subjects. Am Rev Respir Dis 1987;135:554-6.

14 Gianiorio P, Bonavia M, Crimi E, Lantero S, Crimi P, Rossi $\mathrm{GA}$, et al. Bronchial responsiveness is not increased by bronchoalveolar and bronchial lavage performed after allergen challenge. Am Rev Respir Dis 1991;143:105-8.
15 Chetta A, Foresi A, Bertorelli G, Pesci A, Olivieri D. Lung function and bronchial responsiveness after bronchofunction and bronchial responsiveness after bronchopremedication in stable asthmatic subjects. Chest 1992; premedication

16 Kelly C, Hendrick D, Walters $H$. The effects of bronchoalveolar lavage on bronchial responsiveness in patients with airflow obstruction. Chest 1988;93:325-8.

17 Robinson DS, Bentley AM, Hartnell A, Kay AB, Durham SR. Activated memory T helper cells in bronchoalveolar lavage fluid from patients with atopic asthma: relation to asthma symptoms, lung function, and bronchial responsiveness. Thorax 1993;48:26-32.

18 Bentley AM, Qiu Meng, Robinson DS, Hamid Q, Kay $\mathrm{AB}$, Durham SR. Increases in activated T lymphocytes, eosinophils, and cytokine mRNA expression for interleukin-5 and granulocyte/macrophage colony-stimulating leukin- 5 and granulocyte/macrophage colony-stimulating
factor in bronchial biopsies after allergen inhalation challenge in atopic asthmatics. Am 7 Respir Cell Mol Biol 1993; lenge in ator 